\title{
Stellar spectra analysis of giant stars: ARCTURUS
}

\author{
Maria $\operatorname{Rah}^{* 1,2}$ \\ ${ }^{1}$ National Astronomical Observatories and Key Laboratory of Computational Astrophysics, Chinese Academy of Sciences, 20A \\ Datun Rd., Chaoyang District, Beijing 100101, China \\ ${ }^{2}$ Research Institute for Astronomy and Astrophysics of Maragha, Iran
}

\begin{abstract}
In this study, we analyzed the evolved red giant ARCTURUS using high-resolution spectroscopy that was taken by HARPS. The other names of this star is $\alpha$ Boo - Arcturus - HR $5340-$ HD 124897 - HIP 69673. This evolved $(\log g=1.66 \mathrm{dex})$ star shows low metallicity nature $([\mathrm{Fe} / \mathrm{H}]=$ -0.52) which could be employed to study the chemical evolution of the early universe.
\end{abstract}

Keywords: stars: abundances-stars: atmospheres-stars

\section{Introduction}

The fourth brightest star in the Northern Hemisphere Arcturus will die as a white dwarf. This constellation Boötes member has a stellar mass of $1.08 \mathrm{M}_{\odot}$, luminosity in about $\sim 170 \mathrm{~L}_{\odot}$, and age of 7.1 Gyr (Mardini et al., 2019a,b, Masda et al., 2019, Taani et al., 2019b,c). Astronomers believe that Arcturus show high enhancements in the $\alpha$ elements (e.g., C, O, Ne, Mg, Si) relative to iron $([\alpha / \mathrm{Fe}]>+0.4$ dex $)$. This peculiarity in Arcturus atmospheric chemical abundances suggests that it could be a direct descendant of the Population III stars. Therefore, detailed chemical abundances analysis of this astrophysical interesting object might help to constraint it origin (Abdusalam et al., 2020, Mardini. et al., 2019c, Mardini et al., 2020, Placco et al., 2020, Taani et al., 2019a, 2020).

\section{Analysis and some results}

Firstly, we downloaded the archived spectrum HARPS. This spectrum has very high resolution $(\mathrm{R}=$ $40,000)$, signal-to-noise ratio ( $\mathrm{S} / \mathrm{N}=600$ at $4500 \AA)$, and wide wavelength coverage $(\lambda=4100-6000 \AA)$. Taking advantage of this spectrum, we derived the basic stellar parameters: effective temperature (Teff $=4286 \pm 30)$, surface gravity $(\log g=1.66 \pm 0.05)$ and metallicity $([\mathrm{Fe} / \mathrm{H}]=-0.52 \pm 0.04)$ using ATLAS_SYNTHE software. In the second step, we have modeled our target star for which you can find the comparison of the final model with the observational flux in Figure 1.

We then have plotted the $\mathrm{H}_{\alpha}$ line for the models which are different just in one basic stellar parameter, to show the modifications of the models in terms of these changes. As you can see in Figure 2, we have shown the modifications of metallicity versus the temperature in three models, and the effects of temperature and the gravity on the depth of lines.

In the next steps, we plan to provide an accurate atmospheric abundance for 17 light elements; from Oxygen to Zinc. We expect that ARCTURUS atmospheric chemical composition should be consistent with its kinematics (thick-disk star). Collectively, this study tries to improve our understanding of ARCTURUS atmospheric chemical composition that is more important as a differential analysis scale. These parameters and the chemical abundances are typical for the study of the nature and structure of our Galaxy; having accurate stellar parameters and chemical composition of red giant stars, provide useful information about the astrophysical condition that lead to the born of these stars.

*mariarah.astro@gmail.com 


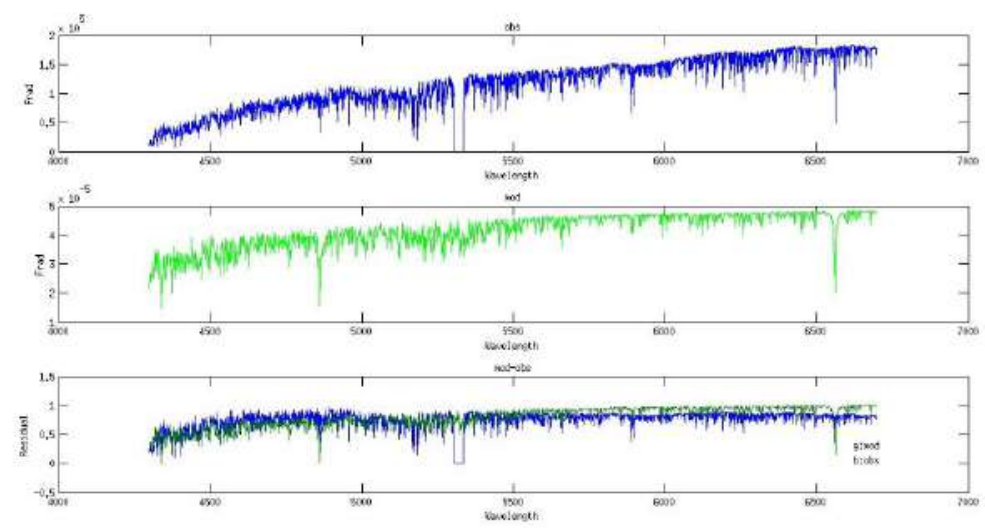

Figure 1. The Comparison of the final model with the observational flux
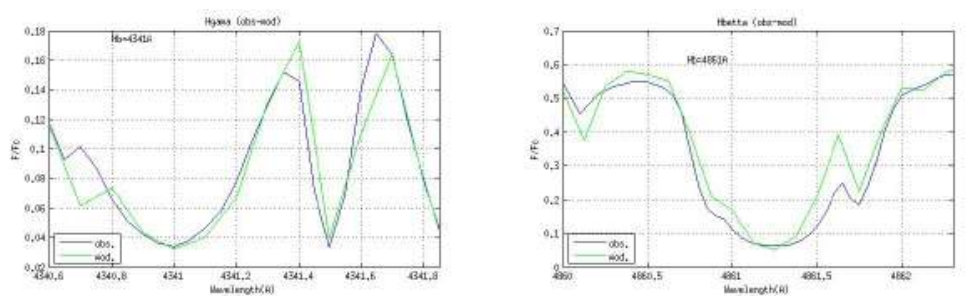

Figure 2. An illustrative example of the effect of modifying metallicity and temperature. These effects can be seen by the depth of lines.

\section{References}

Abdusalam K., Ablimit I., Hashim P., Lü G. L., Mardini M. K., Wang Z. J., 2020, Astrophys. J., 902, 125

Mardini M. K., et al., 2019a, The Astrophysical Journal, 875, 89

Mardini M. K., Placco V. M., Taani A., Li H., Zhao G., 2019b, The Astrophysical Journal, 882, 27

Mardini. M. K., Ershiadat N., Al-Wardat M. A., Taani A. A., Özdemi S., Al-Naimiy H., Khasawneh A., 2019c, in Journal of Physics Conference Series. p. 012024 (arXiv: 1904.09608), doi:10.1088/1742$6596 / 1258 / 1 / 012024$

Mardini M. K., et al., 2020, arXiv e-prints, p. arXiv:2009.12142

Masda S. G., Docobo J. A., Hussein A. M., Mardini M. K., Al-Ameryeen H. A., Campo P. P., Khan A. R., Pathan J. M., 2019, Astrophysical Bulletin, 74, 464

Placco V. M., Santucci R. M., Yuan Z., Mardini M. K., Holmbeck E. M., Wang 2020, Astrophys. J., 897, 78

Taani A., Karino S., Song L., Al-Wardat M., Khasawneh A., Mardini M. K., 2019a, Research in Astronomy and Astrophysics, 19, 012

Taani A., Abushattal A., Mardini M. K., 2019b, Astronomische Nachrichten, 340, 847

Taani A., Karino S., Song L., Mardini M., Al-Wardat M., Abushattal A., Khasawneh A., Al-Naimiy H., 2019c, in Journal of Physics Conference Series. p. 012029, doi:10.1088/1742-6596/1258/1/012029

Taani A., Khasawneh A., Mardini M., Abushattal A., Al-Wardat M., 2020, arXiv e-prints, p. arXiv:2002.03011 